In Theorem Proving in Higher Order Logics (TPHOLs), volume 3223 of Lecture Notes in Computer Science, pages 257-270. Springer, 2004.

\title{
Abstractions for Fault-Tolerant Distributed System Verification
}

\author{
Lee Pike ${ }^{1}$, Jeffrey Maddalon ${ }^{1}$, Paul Miner ${ }^{1}$, and Alfons Geser ${ }^{2}$ \\ 1 Formal Methods Group \\ NASA Langley Research Center \\ M/S 130, Hampton, VA 23681-2199 \\ \{lee.s.pike, j.m.maddalon, paul.s.miner\}@nasa.gov \\ 2 National Institute of Aerospace \\ 144 Research Drive, Hampton, VA 23666 \\ geser@nianet.org
}

\begin{abstract}
Four kinds of abstraction for the design and analysis of faulttolerant distributed systems are discussed. These abstractions concern system messages, faults, fault-masking voting, and communication. The abstractions are formalized in higher-order logic, and are intended to facilitate specifying and verifying such systems in higher-order theoremprovers.
\end{abstract}

\section{Introduction}

In recent years, we have seen tremendous growth in the development of embedded computer systems with critical safety requirements [10, 12], and there is no expectation that this trend will abate. For instance, steerby-wire systems are currently being pursued [11]. To withstand faulty behavior, safety-critical systems have traditionally employed analog backup systems in case the digital system fails; however, many new "by-wire" systems have no analog backup. Instead, they rely on integrated digital fault-tolerance.

Due to their complexity and safety-critical uses, fault-tolerant embedded systems require the greatest assurance of design correctness. One means by which a design can be shown correct is formal methods. Formal methods are especially warranted if we recall that published and peer-reviewed informal proofs-of-correctness of seemingly simple faulttolerant algorithms have been incorrect [16]. Here, we focus on formal methods involving higher-order theorem-provers.

Although many fault-tolerant distributed systems and algorithms have been specified and verified, the abstractions used have often been adhoc and system-specific. Developing appropriate abstractions is often the most difficult and time-consuming part of formal methods [26]. We present these abstractions to systematize and facilitate the practice of abstraction.

The abstractions presented are in the spirit of abstractions of digital hardware developed by Thomas Melham [19, 18]. They are intended to make specifications and their proofs of correctness less tedious [14], less 
error-prone, and more uniform. Although the abstractions we describe are quite general, we intend for them to be accessible to the working verification engineer.

These abstractions are the outcome of the on-going project, "Scalable Processor-Independent Design for Electromagnetic Resilience" (SPIDER), at NASA's Langley Research Center and at the National Institute of Aerospace [29]. SPIDER is the basis of an FAA study exploring the use of formal methods, especially theorem-proving, in avionics certification. One of the project goals is to specify and verify the Reliable Optical Bus (ROBUS), a state-of-the-art fault-tolerant communications bus [28, 21]. The abstractions have proved useful in this project, and in fact are the basis of a generalized fault-tolerant library of PVS theories mentioned in Sect. 8 .

The structure of our paper is as follows. We discuss fault-tolerant distributed systems in Sect. 2. Section 3 gives an overview of the four abstractions presented in this paper. Sections 4 through 7 explain these abstractions. Each section presents an abstraction, and then the abstraction is formalized in higher-order logic. We provide some concluding remarks and point toward future work in the final section.

\section{Fault-Tolerant Distributed Systems}

Introductory material on the foundations of distributed systems and algorithms can be found in Lynch [17]. Some examples of systems that have fault-tolerant distributed implementations are databases, operating systems, communication busses, file systems, and server groups $[3$, $28,2]$.

A distributed system is modeled as a graph with directed edges. Vertices are called processes. Directed edges are called communication channels (or simply channels). If channel $c$ points from process $p$ to process $p^{\prime}$, then $p$ can send messages over $c$ to $p^{\prime}$, and $p^{\prime}$ can receive messages over $c$ from $p$. In this context, $p$ is the sending process (or sender) and $p^{\prime}$ is the receiving process (or receiver). Channels may point from a process to itself. In addition to sending and receiving messages, processes may perform local computation.

A fault-tolerant system is one that continues to provide the required functionality in the presense of faults. One way to implement a faulttolerant system is to use a distributed collection of processes such that a fault that affects one process will not adversely affect the whole system's functionality. This type of system is referred to as a fault-tolerant distributed system.

\section{Four Kinds of Abstraction}

We introduce four fundamental abstractions in the domain of faulttolerant distributed systems. Message Abstractions address the correctness of individual messages sent and received. Fault Abstractions address the kinds of faults possible as well as their effects in the system. FaultMasking Abstractions address the kinds of local computations processes 
make to mask faults. Finally, Communication Abstractions address the kinds of data communicated and the properties required for communication to succeed in the presence of faults.

Our formal expressions are stated in the language of higher-order functions: variables can range over functions, and functions can take other functions as arguments. Furthermore, we use uninterpreted functions (i.e., functions with no defining body) that act as constants when applied to their arguments. Curried functions and lambda abstraction are also used. For a brief overview of higher-order logic from a practitioner's perspective, see, for example, Melham [19] or the PVS language reference [9]. A small datatype, fully explained in Sect. 4, is also used.

The abstractions have been formalized in the Prototype Verification System (PVS), a popular interactive industrial-strength theorem proving system $[22,8]$. They are available at NASA [24].

\section{Abstracting Messages}

\subsection{Abstraction}

Messages communicated in a distributed system are abstracted according to their correctness. We distinguish between benign messages and accepted messages. The former are messages that a non-faulty receiving process recognizes as incorrect; the latter are messages that a non-faulty receiving process does not recognize as incorrect. Note that an accepted message may be incorrect: the receiving process just does not detect that the message is incorrect.

Benign messages abstract various sorts of misbehavior. A message that is sufficiently garbled during transmission may be caught by an errorchecking code [7] and deemed benign. Benign messages also abstract the absence of a message: a receiver expecting a message but detecting the absence of one takes this to be the 'reception' of a benign message. In synchronized systems with global communication schedules, they abstract messages sent and received at unscheduled times.

\subsection{Formalization}

Let the set $M S G$ be a set of messages of a given type. $M S G$ is the base set of elements over which the datatype is defined. The set of all possible datatype elements is denoted by $A B S T R A C T_{-} M S G[M S G]$.

The datatype has two constructors, accepted_msg and benign_msg. The former takes an element $m \in M S G$ and creates the datatype element accepted_msg $[\mathrm{m}]$. The constructor also has an associated extractor value such that

$$
\text { value(accepted_msg }[m])=m \text {. }
$$

The other constructor, benign_msg, is a constant datatype element; it is a constructor with no arguments. All benign messages are abstracted as a single message; thus, the abstracted incorrect message cannot be recovered. Finally, we define two recognizers, accepted_msg? and benign_msg? 
with the following definitions. Let $a \in A B S T R A C T_{-} M S G[M S G]$.

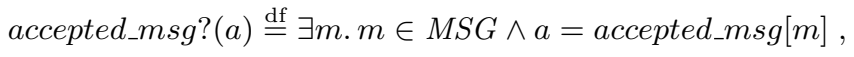

and

$$
\text { benign_msg?(a) } \stackrel{\mathrm{df}}{=} a=\text { benign_msg . }
$$

We summarize this datatype in Fig. 1. Let $m \in M S G$.

\begin{tabular}{r|c|l} 
Constructors & Extractors & Recognizers \\
\hline accepted_msg $[m]$ & value & accepted_msg? \\
benign_msg & none & benign_msg?
\end{tabular}

Fig. 1. Abstract Messages Datatype

\section{$5 \quad$ Abstracting Faults}

There are two closely related abstractions with respect to faults. The first abstraction, error types, partitions the possible locations of faults. The second abstraction, fault types, partitions faults according to the manifestation of the errors caused by the faults. ${ }^{3}$

\subsection{Abstracting Error Types}

Picking the right level of abstraction and the right components to which faults should be attributed is a modeling issue that has been handled in many different ways. We think this is a particularly good example of the extent to which modeling choices can affect specification and proof efficacy.

Both processes and channels can suffer faults [17], but reasoning about process and channel faults together is tedious. Fortunately, such reasoning is redundant - channel faults can be abstracted as process faults. A channel between a sending process and a receiving process can be abstracted as being an extension either of the sender or of the receiver. For instance, a lossy channel abstracted as an extension of the sender is modeled as a process failing to send messages.

Even if we abstract all faults to ones affecting processes and not channels, we are left with the task of abstracting how the functionality of a process - sending, receiving, or computing - is degraded. One possibility is to consider a process as an indivisible unit so that a fault affecting one of its functions is abstracted as affecting its other functions, too. Another possibility is to abstract all faults to ones affecting a process' ability to send and receive messages as in $[27,23]$. Finally, models implicit in $[5,16]$

${ }^{3}$ An error is "that part of the system state which is liable to lead to subsequent failure," while a fault is "the adjudged or hypothesized cause of an error" [15]. 
abstract process faults as being ones affecting only a process' ability to send messages. So even if a fault affects a process' ability to receive messages or compute, the fault is abstractly propagated to a fault affecting the process' ability to send messages.

All three models above are conservative, i.e., the abstraction of a fault is at least as severe as the fault. This is certainly true of the first model in which the whole process is considered to be degraded by any fault, and it is true for the second model, too. Even though it is assumed that a process can always compute correctly, its computed values are inconsequential if it can neither receive nor send correct values. As for the third model, the same reasoning applies - even if a faulty process can receive messages and compute correctly, it cannot send its computations to other processes.

The model we choose is one in which all faults are abstracted to be ones degrading send functionality, and in which channels are abstracted as belonging to the sending process. There are two principal advantages to this model, both of which lead to simpler specifications and proofs. First, the model allows us to disregard faults when reasoning about the ability of processes to receive and compute messages. Second, whether a message is successfully communicated is determined solely by a process' send functionality; the faultiness of receivers need not be considered.

\subsection{Abstracting Fault Types}

Faults result from innumerable occurrences including physical damage, electromagnetic interference, and "slightly-out-of-spec" communication [4]. We collect these fault occurrences into fault types according to their effects in the system.

We adopt the hybrid fault model of Thambidurai and Park [30]. A process is called benign, or manifest, if it sends only benign messages, as described in Sect. 4. A process is called symmetric if it sends every receiver the same message, but these messages may be incorrect. A process is called asymmetric, or Byzantine [13], if it sends different messages to different receivers. All non-faulty processes are also said to be good.

Other fault models exist that provide more or less detail than the hybrid fault model above. The least detailed fault model is to assume the worst case scenario, that all faults are asymmetric. The fault model developed by Azadmanesh and Kieckhafer [1] is an example of a more refined model. All such fault models are consistent with the other abstractions in this paper.

\subsection{Formalization}

We begin by formalizing fault types. Let $S$ and $R$ be sets of processes sending and receiving messages, respectively, in a round of communication. Let asym, sym, ben, and good be constants representing the fault types asymmetric, symmetric, benign, and good, respectively.

As mentioned, we abstract all faults to ones that affect a process' ability to send messages. To model this formally, we construct a function modeling a process sending a message to a receiver. The range of the function is 
the set of abstract messages, elements of the datatype defined in Sect. 4. As explained, $M S G$ is a set of messages, and $A B S T R A C T \_M S G[M S G]$ is the set of datatype elements parameterized by $M S G$. Let $s \in S$ and $r \in R$ be a sending and receiving process, respectively. Let msg_map be a function from senders to the message they intend to send, and let sender_status be a function mapping senders to their fault partition. The function outputs the abstract message received by $r$ from $s$.

$$
\begin{aligned}
& \operatorname{send}\left(m s g \_m a p, \text { sender_status, } s, r\right) \stackrel{\mathrm{df}}{=}
\end{aligned}
$$

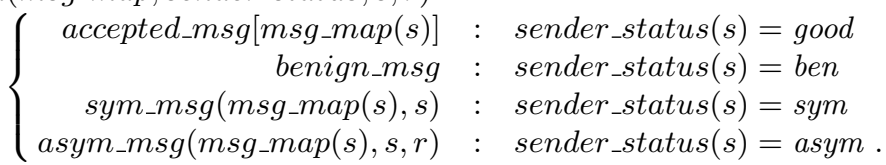

If $s$ is good, then $r$ receives an accepted abstract message, defined in Sect. 4, from $s$. If $s$ is benign, then $r$ receives a benign message. In the last two cases - in which $s$ suffers a symmetric or asymmetric fault - uninterpreted functions are returned. Applied to their arguments, sym_msg and asym_msg are uninterpreted constants of the abstract message datatype defined in Sect. 4. The function asym_msg models a process suffering an asymmetric fault by taking the receiver as an argument: for receivers $r$ and $r^{\prime}$, asym_msg(msg_map $\left.(s), s, r\right)$ is not necessarily equal to asym_msg $\left(m s g \_m a p(s), s, r^{\prime}\right)$. On the other hand, the function $s y m \_m s g$ does not take a receiver as an argument, so all receivers receive the same arbitrary abstract message from a particular sender.

\section{$6 \quad$ Abstracting Fault-Masking}

\subsection{Abstraction}

Some of the information a process receives in a distributed system may be incorrect due to the existence of faults as described in Sect. 5. A process must have a means to mask incorrect information generated by faulty processes. Two of the most well-known are (variants of) a majority vote or a middle-value selection, as defined in the following paragraph. These functions are similar enough to abstract them as a single fault-masking function.

A majority vote returns the majority value of some multiset (i.e., a set in which repetition of values is allowed), and a default value if no majority exists. A middle-value selection takes the middle value of a linearlyordered multiset if the cardinality of the multiset is odd. If the cardinality is an even integer $n$, then the natural choices are to compute one of (1) the value at index $\lfloor n / 2\rfloor,(2)$ the value at index $\lceil n / 2\rceil$, or (3) the average of the two values from (1) and (2). Of course, these options may yield different values; in fact, (3) may yield a value not present in the multiset. For example, for the multiset $\{1,1,2,2,2,2\}$, the majority value is 2 , and the middle-value selection is also 2 for any of the three ways to compute the middle-value selection. For any multiset that can be linearly-ordered, if a majority value exists, then the majority value is equal to the middlevalue selection (for any of the three ways to compute it mentioned above). 
The benefit of this abstraction is that we can define a single fault-masking function (we call it a fault-masking vote) that can be implemented as either a majority vote or a middle-value selection (provided the data over which the function is applied is linearly-ordered).

This allows us to model what are usually considered to be quite distinct fault-tolerant distributed algorithms uniformly. Concretely, this abstraction, coupled with the other abstractions described in this paper, allow certain clock synchronization algorithms (which usually depend on a middle-value selection) and algorithms in the spirit of an Oral Messages algorithm $[13,16]$ (which usually depend on a majority vote) to share the same underlying models [20].

\subsection{Formalization}

The formalization we describe models a majority vote and a middlevalue selection over a multiset. A small lemma stating their equivalence follows. Definitions of standard and minor functions are omitted.

Based on the NASA Langley Research Center PVS Bags Library [6], a multiset is formalized as a function from values to the natural numbers that determines how many times a value appears in the multiset (values not present are mapped to 0 ). Thus, let $V$ be a nonempty finite set of values $^{4}$, and let $m s: V \rightarrow \mathbb{N}$ be a multiset.

To define a majority vote, we define the cardinality of a multiset $\mathrm{ms}$ to be the summation of value-instances in it:

$$
|m s| \stackrel{\mathrm{df}}{=} \sum_{v \in V} m s(v) .
$$

The function $m a j \_s e t$ takes a multiset $m s$ and returns the set of majority values in it.

$$
m a j_{s} s e t(m s) \stackrel{\text { df }}{=}\{v|2 \times m s(v)>| m s \mid\} .
$$

This set is empty if no majority value exists, or it is a singleton set. Thus, we define majority to be a function returning the special constant no_majority if no majority value exists and the single majority value otherwise.

$$
\operatorname{majority}(m s) \stackrel{\text { df }}{=} \begin{cases}\text { no_majority } & : \text { maj_set }(m s)=\emptyset \\ \epsilon(\text { maj_set }(m s)) & : \text { otherwise }\end{cases}
$$

The function $\epsilon$ is the choice operator that takes a set and returns an arbitrary value in the set if the set is nonempty. Otherwise, an arbitrary value of the same type as the elements in the set is returned [19].

Now we formalize a middle-value selection. Let $V$ have the linear order

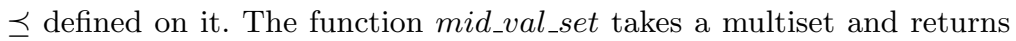
the set of values at index $\lceil n / 2\rceil$ when the values are ordered from least to

${ }^{4}$ If $V$ is finite, then multisets are finite. Fault-masking votes can only be taken over finite multisets. 
greatest (we arbitrarily choose this implementation). The set is always a singleton set.

$$
\begin{aligned}
& \text { mid_val_set }(m s) \stackrel{\text { df }}{=} \\
& \left\{v \mid \begin{array}{l}
2 \times \mid \text { lower_filter }(m s, v)|>| m s \mid \wedge \\
2 \times \mid \text { upper_filter }(m s, v)|\geq| m s \mid
\end{array}\right\} .
\end{aligned}
$$

The function lower_filter filters out all of the values of $m s$ that are less than or equal to $v$, and upper_filter filters out the values greater than or equal to $v$. The function lower_filter is defined as follows:

$$
\text { lower_filter }(m s, v) \stackrel{\mathrm{df}}{=} \lambda i .\left\{\begin{aligned}
m s(i) & : \quad i \preceq v \\
0 & : \text { otherwise . }
\end{aligned}\right.
$$

Similarly,

$$
\text { upper_filter }(m s, v) \stackrel{\text { df }}{=} \lambda i .\left\{\begin{aligned}
m s(i) & : \quad v \preceq i \\
0 & : \text { otherwise . }
\end{aligned}\right.
$$

The relation mid_val_set $(m s)$ is guaranteed to be a singleton set, so using the function $\epsilon$ mentioned above, we can define middle_value to return the middle value of a multiset:

$$
\text { middle_value }(m s) \stackrel{\text { df }}{=} \epsilon(\text { mid_val_set }(m s)) \text {. }
$$

The following theorem results.

Theorem 1 (Middle Value is Majority). majority $(\mathrm{ms}) \neq$ no_majority implies middle_value $(\mathrm{ms})=$ majority $(\mathrm{ms})$.

\section{Abstracting Communication}

We identify two abstractions with respect to communication. First, we abstract the kinds of data communicated. Second, we identify the fundamental conditions that must hold for communication to succeed.

\subsection{Abstracting Kinds of Communication}

Some kinds of information can be modelled by real valued, uniformly continuous functions. Intuitively, a function is uniformly continuous if small changes in its argument produce small changes in its result; see e.g., Rosenlicht [25]. For example, the values of analog clocks and of thermometers vary with time, and the rate of change is bounded. In a distributed system, a process may sample such a function, i.e., determine an approximation of the function's value for a given input. We call such functions inexact functions and the communication of their values inexact communication. We call discrete functions, such as an array sorting algorithm, exact functions and communication involving them exact communication. 


\subsection{Abstracting Communication Conditions}

Communication in a fault-tolerant distributed system is successful if $v a$ lidity and agreement hold. For exact communication, their general forms are:

Exact Validity: A good receiver's fault-masking vote is equal to the value of the function good processes compute.

Exact Agreement: All good processes have equal fault-masking votes. For inexact communication we have similar conditions:

Inexact Validity: A good receiver's fault-masking vote is bounded above and below by the samples from good processes, up to a small error margin.

Inexact Agreement: All good processes differ in their fault-masking votes by at most a small margin of error.

A validity property can thus be understood as an agreement between senders and receivers, whereas an agreement property is an agreement between the receivers. For lack of space, we limit our presentation to guaranteeing validity. Agreement is treated similarly, and complete PVS formalizations and proofs for both are located at NASA [24].

We distinguish between a functional model and a relational model of communication. In the former, communication is modeled computationally (e.g., using functions like send from Sect. 5). In the latter more abstract model, conditions on communication are stated such that if they hold, communication succeeds. This section presents a relational model of communication.

We specifically present conditions that guarantee validity holds after a single broadcast communication round in which each process in a set of senders sends messages to each process in a set of receivers (a degenerate case is when these are singleton sets modeling point-to-point communication between a single sender and receiver). A functional model of a specific communication protocol can be shown to satisfy these conditions through step-wise refinement.

First we describe how a single round of exact communication satisfies exact validity, provided that the three conditions Majority Good, Exact Function, and Function Agreement hold. The three conditions state, respectively, that the majority of the values over which a vote is taken come from good senders, that good senders compute functions exactly (i.e., there is no approximation in sampling an exact function), and that every good sender computes the same function.

For a single round of inexact communication, we have inexact validity if the two conditions Majority Good and Inexact Function hold. Majority Good is the same as above. The Inexact Function condition bounds the variance allowed between the sample of an inexact function computed by a good process for a given input and the actual value of the function for that input. That is, let $\varepsilon_{1}$ and $\varepsilon_{\mathrm{u}}$ be small positive constants representing the lower and upper variation, respectively, allowed between an inexact function $f$ and potential samples of it as depicted in Fig. 2. The sample computed by a good process is bounded by $f-\varepsilon_{1}$ and $f+\varepsilon_{\mathrm{u}}$. We do not present an analog to the Function Agreement condition in the inexact case since processes often compute and vote over slightly different functions. For example, each process might possess a distinct local sensor 


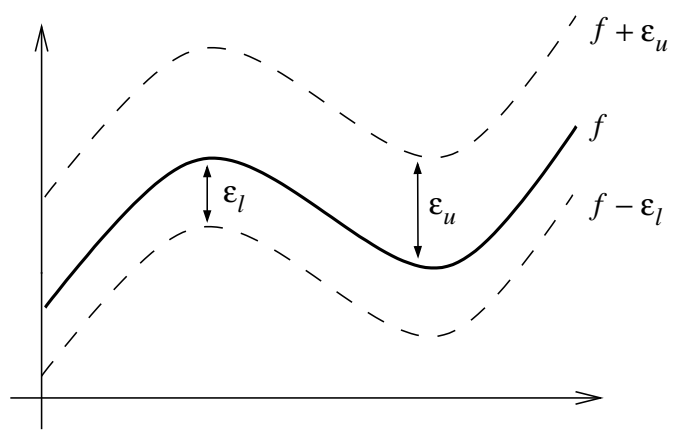

Fig. 2. The Inexact Function Condition for Inexact Communication

that it samples. It is assumed, however, that the functions are related, e.g., each sensor measures the same aspect of the environment.

Clock synchronization [17] is an important case of inexact communication. Clocks distributed in the system need to be synchronized in order to avoid drifting too far apart. In this case, sampling a local clock yields an approximation of time.

\subsection{Formalization for Exact Communication}

First we present the model of a round of exact communication. For a single round of communication, let $S$ be the set of senders sending in that round. Let good_sender $\subseteq S$ be a subset of senders that are good. This set can change as processes become faulty and are repaired, so we treat it as a variable rather than a constant. For an arbitrary receiver, ${ }^{5}$ let eligible_senders $\subseteq S$ be the set of senders trusted by the receiver (we assume that receivers trust all good senders). Then the condition Majority Good is defined

$$
\begin{aligned}
& \text { majority_good }(\text { good_senders, eligible_senders }) \stackrel{\text { df }}{=} \\
& 2 \times \mid \text { good_senders }|>| \text { eligible_senders } \mid \wedge \\
& \text { good_senders } \subseteq \text { eligible_senders } .
\end{aligned}
$$

This stipulates that a majority of the senders in eligible_senders are in good_senders.

Next we describe the values sent and received. Let $M S G$ be the range of the function computed - these are the messages communicated. The variable ideal : $S \rightarrow M S G$ maps a sender to the exact value of a function to be computed by the sender, for a given input. This frees us from representing the particular function computed. Similarly, actual $: S \rightarrow$

\footnotetext{
5 The receiver can be any receiver, good or faulty. The abstractions described in Sect. 5 allow us to ignore the fault status of receivers in formal analysis.
} 
$M S G$ maps a sender to the value that sender actually computes for the same function and input. Good senders compute exact functions exactly:

$$
\begin{aligned}
& \text { exact_function }(\text { good_senders, ideal, actual) } \stackrel{\mathrm{df}}{=} \\
& \forall s . s \in \text { good_senders } \Longrightarrow \text { ideal }(s)=\operatorname{actual}(s) .
\end{aligned}
$$

Function Agreement states that the functions computed by any two good senders is the same (i.e., they send the same messages).

function_agreement $($ good_senders, ideal) $\stackrel{\mathrm{df}}{=}$

$$
\forall s_{1}, s_{2} . s_{1} \in \text { good_senders } \wedge s_{2} \in \text { good_senders } \Longrightarrow \operatorname{ideal}\left(s_{1}\right)=i d e a l\left(s_{2}\right) \text {. }
$$

Before stating the validity result, we must take care of a technical detail with respect to forming the multiset of messages over which a receiver takes a fault-masking vote. For an arbitrary receiver, let the function make_bag take as arguments a nonempty set eligible_senders and a function mapping senders to the message the receiver gets. It returns a multiset of received messages from senders in eligible_senders.

$$
\begin{aligned}
& \text { make_bag }(\text { eligible_senders, actual }) \stackrel{\mathrm{df}}{=} \\
& \lambda v . \mid\{s \mid s \in \text { eligible_senders } \wedge \text { actual }(s)=v\} \mid .
\end{aligned}
$$

For exact messages, validity is the proposition that for any good sender, the exact value of the function it is to compute is the value computed by the receiver's fault-masking vote. This proposition is defined as follows:

$$
\begin{aligned}
& \text { exact_validity }(\text { eligible_senders,good_senders,ideal,actual }) \stackrel{\mathrm{df}}{=} \\
& \forall s . s \in \text { good_senders } \Longrightarrow \\
& \text { ideal }(s)=\text { majority }(\text { make_bag }(\text { eligible_senders,actual }))
\end{aligned}
$$

We use majority for the fault-masking vote, but middle-value selection is acceptable given Thm. 1. Using majority, the Exact Validity Theorem reads: 
Theorem 2 (Exact Validity).

majority_good(good_senders, eligible_senders) and

exact_function(good_senders, ideal,actual)) and

function_agreement(good_senders,ideal)

implies that

exact_validity(eligible_senders,good_senders, ideal,actual) .

\subsection{Formalization for Inexact Communication}

Next we model a round of inexact communication. The Majority Good condition is formalized the same as for exact communication. To define Inexact Function, we now assume that the elements of $M S G$ have at least the structure of an additive group linearly ordered by $\preceq$. Inexact Function is defined as the conjunction of two conditions, Lower Function Error and Upper Function Error. These two conditions specify, respectively, the maximal negative and positive error between the exact value of an inexact function and a good sender's approximation of the inexact function, for a given input.

$$
\begin{aligned}
& \text { lower_function_error }(\text { good_senders, ideal, actual }) \stackrel{\mathrm{df}}{=} \\
& \forall s . s \in \text { good_senders } \Longrightarrow \operatorname{ideal}(s)-\varepsilon_{1} \preceq \operatorname{actual}(s) \text {; } \\
& \text { upper_function_error(good_senders, ideal, actual) } \stackrel{\mathrm{df}}{=} \\
& \forall s . s \in \text { good_senders } \Longrightarrow \operatorname{actual}(s) \preceq \text { ideal }(s)+\varepsilon_{\mathrm{u}} ; \\
& \text { inexact_function (good_senders, ideal, actual) } \stackrel{\mathrm{df}}{=} \\
& \text { lower_function_error(good_senders, ideal,actual) } \wedge \\
& \text { upper_function_error(good_senders, ideal,actual) . }
\end{aligned}
$$

For inexact communication, validity is the proposition that for a fixed receiver, the value determined by a fault-masking vote is bounded both above and below by the messages received from good senders, modulo error values $\varepsilon_{1}$ and $\varepsilon_{\mathrm{u}}$. Note that each sender may be computing a different inexact function, so the vote window depends on both the functions computed as well as the errors in approximating them. This is illustrated in Fig. 3, where $s_{1}$ and $s_{2}$ are good senders.

$$
\begin{aligned}
& \text { inexact_validity }(\text { eligible_senders,good_senders, ideal }, \text { actual }) \stackrel{\mathrm{df}}{=} \\
& \exists s_{1} . s_{1} \in \text { good_senders } \wedge \\
& \quad \text { ideal }\left(s_{1}\right)-\varepsilon_{1} \preceq \text { middle_value }(\text { make_bag }(\text { eligible_senders, actual })) \wedge \\
& \exists s_{2} . s_{2} \in \text { good_senders } \wedge \\
& \quad \text { middle_value }(\text { make_bag }(\text { eligible_senders }, \text { actual })) \preceq i d e a l\left(s_{2}\right)+\varepsilon_{\mathrm{u}} .
\end{aligned}
$$

The Inexact Validity Theorem then reads:

Theorem 3 (Inexact Validity).

majority_good(good_senders, eligible_senders) and 


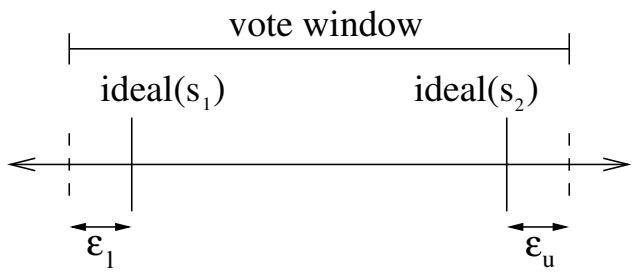

Fig. 3. Inexact Validity

inexact_function(good_senders, ideal,actual)

implies that

inexact_validity(eligible_senders,good_senders, ideal,actual) .

\section{Conclusion}

This paper presents, in the language of higher-order logic, four kinds of abstractions for fault-tolerant distributed systems. These abstractions pertain to messages, faults, fault-masking, and communication. We believe that they abstract a wide-variety of fault-tolerant distributed systems.

Other useful abstractions have been developed, too. For example, Rushby demonstrates how to derive a time-triggered system from the specification of the system as a (synchronous) functional program [27]. This work has been used in the specification and verification of the Time-Triggered Architecture [23]. With respect to these works, the abstractions we give systematize specification and verification at the level of the functional programs.

Our abstractions have proved their merit in an industrial-scale formal specification and verification project. We are sure that similar projects will profit. We are developing a distributed fault-tolerance library as part of the SPIDER project. It is designed to be a generic library of PVS theories that may be used in the specification and verification of a wide variety of fault-tolerant distributed systems. The abstractions described in this paper form the backbone of the library.

\section{Acknowledgments}

We would like to thank Victor Carreño and Kristen Rozier as well as our anonymous referees for helpful comments.

\section{References}

1. Mohammad H. Azadmanesh and Roger M. Kieckhafer. Exploiting omissive faults in synchronous approximate agreement. IEEE Transactions on Computers, 49(10):1031-1042, 2000. 
2. Miguel Castro and Barbara Liskov. Practical Byzantine fault tolerance. In ACM Proceedings: Operating Systems Design and Implementation (OSDI), pages 173-186, February 1999.

3. Flaviu Cristian. Understanding fault-tolerant distributed systems. Communications of the ACM, 34(2), February 1991.

4. Kevin Driscoll, Brendan Hall, Håkan Sivencrona, and Phil Zumsteg. Byzantine fault tolerance, from theory to reality. In G. Goos, J. Hartmanis, and J. van Leeuwen, editors, Computer Safety, Reliability, and Security, Lecture Notes in Computer Science, pages 235-248. The 22nd International Conference on Computer Safety, Reliability and Security SAFECOMP, Springer-Verlag Heidelberg, September 2003 .

5. Alfons Geser and Paul Miner. A formal correctness proof of the SPIDER diagnosis protocol. Technical Report 2002-211736, NASA Langley Research Center, Hampton, Virginia, August 2002. Technical Report contains the Track B proceedings from Theorem Proving in Higher Order Logics (TPHOLSs).

6. NASA LaRC Formal Methods Group. NASA Langley PVS libraries. Available at http://shemesh.larc.nasa.gov/fm/ftp/ larc/PVS-library/pvslib.html.

7. Gerard J. Holzmann. Design and Validation of Computer Protocols. Prentice Hall, 1991.

8. SRI International. PVS homepage. Available at http://pvs.csl. sri.com/.

9. SRI International. PVS language reference, version 2.4. Available at http://pvs.csl.sri.com/manuals.html, December 2001.

10. Steven D. Johnson. Formal methods in embedded design. Computer, pages 104-106, Novemeber 2003.

11. Philip Koopman, editor. Critical Embedded Automotive Networks, volume 22-4 of IEEE Micro. IEEE Computer Society, July/August 2002.

12. Hermann Kopetz. Real-Time Systems. Kluwer Academic Publishers, 1997.

13. Lamport, Shostak, and Pease. The Byzantine generals problem. ACM Transactions on Programming Languages and Systems, 4:382401, July 1982.

14. Leslie Lamport. Composition: A way to make proofs harder. Lecture Notes in Computer Science, 1536:402-423, 1998.

15. Jean-Claude Laprie. Dependability — its attributes, impairments and means. In B. Randell, J.-C. Laprie, H. Kopetz, and B. Littlewood, editors, Predictability Dependable Computing Systems, ESPRIT Basic Research Series, pages 3-24. Springer, 1995.

16. Patrick Lincoln and John Rushby. The formal verification of an algorithm for interactive consistency under a hybrid fault model. In Costas Courcoubetis, editor, Computer-Aided Verification, CAV'93, volume 697 of Lecture Notes in Computer Science, pages 292-304, Elounda, Greece, June/July 1993. Springer-Verlag.

17. Nancy A. Lynch. Distributed Algorithms. Morgan Kaufmann, 1996.

18. Thomas F. Melham. Abstraction mechanisms for hardware verification. In G. Birtwistle and P.A. Subrahmanyam, editors, VLSI Spec- 
ification, Verification, and Synthesis, pages 129-157, Boston, 1988. Kluwer Academic Publishers.

19. Thomas F. Melham. Higher Order Logic and Hardware Verification. Cambridge Tracts in Theoretical Computer Science. Cambridge University Press, 1993.

20. Paul Miner, Alfons Geser, Lee Pike, and Jeffrey Maddalon. A unified fault-tolerance protocol. In preparation. Available at http://shemesh.larc.nasa.gov/fm/spider/spider_pubs.html, April 2004.

21. Paul S. Miner, Mahyar Malekpour, and Wilfredo Torres-Pomales. Conceptual design of a Reliable Optical BUS (ROBUS). In 21st AIAA/IEEE Digital Avionics Systems Conference DASC, Irvine, CA, October 2002.

22. Sam Owre, John Rushby, Natarajan Shankar, and Friedrich von Henke. Formal verification for fault-tolerant architectures: Prolegomena to the design of PVS. IEEE Transactions on Software Engineering, 21(2):107-125, February 1995.

23. Holger Pfeifer. Formal Analysis of Fault-Tolerant Algorithms in the Time-Triggered Architecture. $\mathrm{PhD}$ thesis, Universität Ulm, 2003. Available at http://www.informatik.uni-ulm.de/ki/ Papers/pfeifer-phd.html.

24. Lee Pike, Jeffrey Maddalon, Paul Miner, and Alfons Geser. PVS specifications and proofs for fault-tolerant distributed system verification. Available at http://shemesh.larc.nasa.gov/fm/spider/ tphols2004/pvs.html, 2004.

25. Maxwell Rosenlicht. Introduction to Analysis. Dover Publications, Inc., 1968.

26. John Rushby. Formal methods and digital systems validation for airborne systems. Technical Report CR-4551, NASA, December 1993.

27. John Rushby. Systematic formal verification for fault-tolerant timetriggered algorithms. IEEE Transactions on Software Engineering, 25(5):651-660, September/October 1999.

28. John Rushby. A comparison of bus architectures for safety-critical embedded systems. Technical report, Computer Science Laboratory, SRI International, Menlo Park, CA, September 2001. Available at http://www.csl.sri.com/ ushby/abstracts/buscompare.

29. SPIDER homepage, NASA Langley Research Center, Formal Methods Team. Available at http://shemesh.larc.nasa.gov/fm/ spider/.

30. Philip Thambidurai and You-Keun Park. Interactive consistency with multiple failure modes. In 7th Reliable Distributed Systems Symposium, pages 93-100, October 1988. 